\title{
Extensions of the RGA Concept to Nonlinear Systems
}

\author{
S. T. Glad \\ Department of Electrical Engineering \\ Linköping University, SE-581 83 Linköping, Sweden \\ WWW: http://www. control.isy.liu.se \\ Email: torkel@isy.liu.se
}

March 16, 2000

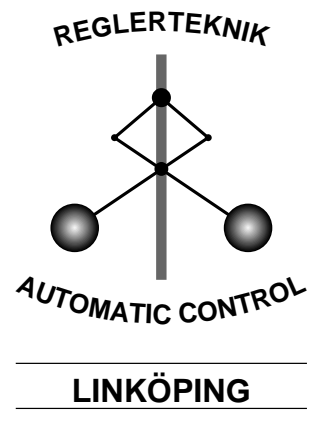

Report no.: LiTH-ISY-R-2229

Presented at ECC99

Technical reports from the Automatic Control group in Linköping are available by anonymous ftp at the address ftp.control.isy.liu.se. This report is contained in the file 2229.pdf. 



\title{
Extensions of the RGA Concept to Nonlinear Systems
}

\author{
S.T. Glad \\ Department of Electrical Engineering \\ Linköping University \\ SE-581 83 Linköping, Sweden \\ e-mail: torkel@isy.liu.se
}

March 16, 2000

Keywords : Nonlinear systems, RGA, decoupling matrix

\begin{abstract}
Extentions of the RGA (relative gain array) technique to nonlinear systems are considered. The steady-state properties are given by an array of nonlinear functions. The high frequency properties are characterized by forming the conventional RGA of the decoupling matrix.
\end{abstract}

\section{Introduction}

The relative gain array (RGA) has been widely used as a measure of the interaction between control loops in multivariable systems, see e. g. [1], [2], [4]. For a linear system with square transfer matrix $G(s)$, the relative gain array is defined as

$$
G_{R G A}=G \cdot * G^{-T}
$$

where ".*" denotes element-wise multiplication of the matrices, and $-T$ denotes the transpose of the inverse. Often the matrices are evaluated at $s=0$ so that the static gains are considered, but it is also possible to look at arbitrary frequencies $s=i \omega$. The element $i, j$ in the RGA array can be interpreted as the gain from input $u_{j}$ to output $y_{i}$ when the other $u_{k}$ are zero ("all other loops open"), divided by the corresponding gain when all other $y_{k}$ are zero ("all other loops have maximally tight control"). It is the purpose of this paper to suggest extensions of the RGA concept to nonlinear systems.

\section{A nonlinear static RGA}

Consider a dynamic system with input $u$ and output $y$, both being $m$-vectors. Assume that for each constant $u$ there exists an equilibrium of the system and a corresponding constant $y$, so that we have

$$
y=H(u)
$$


for some function $H$ from $\mathbb{R}^{m}$ to $\mathbb{R}^{m}$. We consider some reference point $u^{0}$ and define $y^{0}=H\left(u^{0}\right)$. We also define

$$
\phi_{i j}\left(u_{j}\right)=H_{i}\left(u_{1}^{0}, \ldots, u_{j-1}^{0}, u_{j}, u_{j+1}^{0}, \ldots, u_{m}^{0}\right)
$$

where $H_{i}$ denotes the $i$ :th component of $H$. The function $\phi_{i j}$ thus shows how $y_{i}$ depends on $u_{j}$ when all other outputs are kept at the nominal value $u^{0}$. If $H$ has an inverse it is possible to define

$$
\psi_{j i}\left(y_{i}\right)=\left(H^{-1}\right)_{j}\left(y_{1}^{0}, \ldots, y_{i-1}^{0}, y_{i}, y_{i+1}^{0}, \ldots, y_{m}^{0}\right)
$$

which can be interpreted as the input $u_{j}$ which is needed to get the output $y_{i}$, provided all other controls are chosen to keep $y_{k}, k \neq i$ equal to the nominal values $y_{k}^{0}$. It is now possible to define a nonlinear static RGA.

Definition 1 For a steady-state input-output relationship (2), with $H$ invertible, the relative gain array is defined as

$$
H_{i j}^{R G A}=\phi_{i j}\left(\psi_{j i}\left(y_{i}\right)\right)
$$

The relative gain array is thus an $m$ by $m$ array of scalar functions. If, for each $i, H_{i}$ depends only on $u_{i}$ - the perfectly decoupled situation - then

$$
H_{i j}^{R G A}= \begin{cases}y_{i}^{0} & i \neq i \\ y_{i} & i=j\end{cases}
$$

The extent to which $H^{R G A}$ differs from this is thus a measure of the extent of static coupling.

We will now assume that the dynamic system is given by

$$
\dot{x}=f(x, u), \quad y=h(x)
$$

where $f$ and $h$ are continuously differentiable functions.

Proposition 1 Let $x^{0}, u^{0}$ be such that $f\left(x^{0}, u^{0}\right)=0$ and define $y^{0}=h\left(x^{0}\right)$.

1. If $f_{x}\left(x^{0}, u^{0}\right)$ is nonsingular then the function $H$ of (2) is well defined in a neighborhood of $u^{0}$.

2. If, in addition $h_{x}\left(x^{0}\right) f_{x}\left(x^{0}, u^{0}\right)^{-1} f_{u}\left(x^{0}, u^{0}\right)$ is nonsingular, then $H$ is invertible, $i$. e. the relative gain array is well defined in a neighborhood of $y^{0}$. Moreover, the elements of $H^{R G A}$ are differentiable in the same neighborhood.

Proof. From the implicit function theorem it follows that there exists a function $\xi(x, u)$, defined in some neighborhood $U_{1} \subset R^{n+m}$ of $x^{0}, u^{0}$, such that

$$
0=f(\xi(u), u)
$$

Moreover $\xi$ is differentiable with derivative given by

$$
\xi_{x}(u)=-f_{x}(\xi(u), u)^{-1} f_{u}(\xi(u), u)
$$

The function $H$ can now be defined as

$$
H(u)=h(\xi(u))
$$

Since

$$
H_{u}\left(u^{0}\right)=h_{x}\left(y^{0}\right) \xi_{x}\left(u^{0}\right)=-h_{x}\left(y^{0}\right) f_{x}\left(x^{0}, u^{0}\right)^{-1} f_{u}\left(x^{0}, u^{0}\right)
$$

which is nonsingular by assumption, the existence of a differentiable inverse of $H$ close to $y^{0}$ is guaranteed by the implicit function theorem. 
Example 1 Consider a tank system with four tanks whose levels are $x_{1}$ through $x_{4}$. The outputs $y_{1}$ and $y_{2}$ are the levels of tanks 1 and 2 , while the inputs $u_{1}$ and $u_{2}$ are the inflows of tanks 3 and 4 . Tank 3 empties into tank 1 and tank 4 into tank 2. There are also smaller flows from tank 3 into tank 2 and from $u_{2}$ into tank 1 , scaled by parameters $\alpha$ and $\beta$. A simple model is

$$
\begin{aligned}
& \dot{x}_{1}=-\sqrt{x_{1}}+\sqrt{x_{3}}+\alpha u_{2} \\
& \dot{x}_{2}=-\sqrt{x_{2}}+\sqrt{x_{4}}+\beta \sqrt{x_{3}} \\
& \dot{x}_{3}=-(1+\beta) \sqrt{x_{3}}+u_{1} \\
& \dot{x}_{4}=-\sqrt{x_{4}}+u_{2} \\
& y_{1}=x_{1} \\
& y_{2}=x_{2}
\end{aligned}
$$

The relative gain array for $u_{1}^{0}=1+\beta, u_{2}^{0}=1$ (which implies $y_{1}^{0}=(1+\alpha)^{2}$, $\left.y_{2}^{0}=(1+\beta)^{2}\right)$ is

$$
\begin{aligned}
& H_{11}^{R G A}=\left(\alpha \beta+\alpha^{2} \beta-\sqrt{y_{1}}\right)^{2} /(\alpha \beta-1)^{2} \\
& H_{12}^{R G A}=\left(\alpha \beta \sqrt{y_{1}}-1-\alpha\right)^{2} /(\alpha \beta-1)^{2} \\
& H_{21}^{R G A}=\left(\alpha \beta \sqrt{y_{2}}-1-\beta\right)^{2} /(\alpha \beta-1)^{2} \\
& H_{22}^{R G A}=\left(\alpha \beta+\alpha \beta^{2}-\sqrt{y_{2}}\right)^{2} /(\alpha \beta-1)^{2}
\end{aligned}
$$

Note that $\alpha=, \beta=0$, which removes the physical coupling between the two chains of tanks, gives

$$
H^{R G A}=\left[\begin{array}{cc}
y_{1} & 1 \\
1 & y_{2}
\end{array}\right]
$$

in agreement with (6).

\section{General dynamic systems}

It is natural to generalize definition 1 to the dynamic case by replacing $H$ by some mapping

$$
y=\mathcal{H}(u)
$$

where $u$ and $y$ are now regarded as elements in appropriate function spaces and $\mathcal{H}$ is an operator between these function spaces. In analogy with (3) and (4) we can define

$$
\begin{aligned}
\Phi_{i j}\left(u_{j}\right) & =\mathcal{H}_{i}\left(u_{1}^{0}, \ldots, u_{j-1}^{0}, u_{j}, u_{j+1}^{0}, \ldots, u_{m}^{0}\right) \\
\Psi_{j i}\left(y_{i}\right) & \left.=\left(\mathcal{H}^{-1}\right)_{j} s y_{1}^{0}, \ldots, y_{i-1}^{0}, y_{i}, y_{i+1}^{0}, \ldots, y_{m}^{0}\right)
\end{aligned}
$$

provided the inverse exists. The natural definition of the relative gain array would then be

$$
\mathcal{H}_{i j}^{R G A}=\Phi_{i j}\left(\Psi_{j i}\left(y_{i}\right)\right)
$$

This would then be an array of operators between function spaces. To get a more concrete representation we will look at control affine systems. 


\section{Control affine system}

Consider a control affine system

$$
\dot{x}=f(x)+g(x) u, \quad y=h(x)
$$

with $n$ state variables, $m$ control variables and $m$ outputs. For simplicity we assume that $x^{0}=0, u^{0}=0, y^{0}=0$ and that $f(0)=0, h(0)=0$.

The appearance of the inverse in the abstract formula (10) makes it clear that the extension of the relative gain array concept will be closely linked to invertibility theory of control systems, see [3], [6], [7], [5]. We assume that the system has a vector relative degree $r_{1}, \ldots, r_{m}$ at $x_{0}$ and introduce the decoupling matrix

$$
R(x)=\left[\begin{array}{ccc}
L_{g_{1}} L_{f}^{r_{1}-1} h_{1} & \ldots & L_{g_{m}} L_{f}^{r_{1}-1} h_{1} \\
\vdots & & \vdots \\
L_{g_{1}} L_{f}^{r_{m}-1} h_{m} & \ldots & L_{g_{m}} L_{f}^{r_{m}-1} h_{m}
\end{array}\right]
$$

and the vectors

$$
a(x)=\left[\begin{array}{c}
L_{f}^{r_{1}} h_{1} \\
\vdots \\
L_{f}^{r_{m}} h_{m}
\end{array}\right], \quad Y=\left[\begin{array}{c}
y_{1}^{\left(r_{1}\right)} \\
\vdots \\
y_{m}^{\left(r_{m}\right)}
\end{array}\right]
$$

It is possible to introduce new coordinates $\eta$ and $\xi$, where $\xi$ has the components $\xi_{1}^{i}, \ldots, \xi_{r_{i}}^{i}, i=1, \ldots, m$, in such a way that the system description has the following form, [5].

$$
\begin{aligned}
\dot{\xi}_{1}^{k} & =\xi_{2}^{k} \\
& \vdots \\
\dot{\xi}_{r_{k}}^{k} & =a_{k}(\xi, \eta)+\sum R(\xi, \eta)_{k j} u_{j}, \quad k=1, \ldots, m \\
\dot{\eta} & =b(\xi, \eta)+d(\xi, \eta) u \\
y_{k} & =\xi_{1}^{k} \quad k=1, \ldots, m
\end{aligned}
$$

The relation between input and output can compactly be written as

$$
Y=a(\xi, \eta)+R(\xi, \eta) u
$$

Note that the $R$ is nonsingular in a neighborhood of $x^{0}$ since we assume the existence of a vector relative degree.

Proposition 2 For the system (15) the operator $\tilde{y}=\mathcal{H}_{i j}^{R G A}(y)$ of (11) is represented by the following dynamic system.

$$
\begin{aligned}
\dot{\xi}_{1}^{k} & =\xi_{2}^{k} \\
& \vdots \\
\dot{\xi}_{r_{k}}^{k} & =a_{k}(\xi, \eta)+R(\xi, \eta)_{k j} u_{j}, \quad k=1, \ldots, m \\
\dot{\eta} & =b(\xi, \eta)+d(\xi, \eta) u \\
\tilde{y}_{i} & =\xi_{1}^{i}
\end{aligned}
$$


where $u_{j}$ is calculated by

$$
\begin{aligned}
u_{j} & =\left(R^{-1}\left(\xi_{Y i}, \zeta\right)\left(Y_{i}-a\left(\xi_{Y i}, \zeta\right)\right)\right)_{j} \\
\dot{\zeta} & =b\left(\xi_{Y i}, \zeta\right)+d\left(\xi_{Y i}, \zeta\right) R^{-1}\left(\xi_{Y i}, \zeta\right)\left(Y_{i}-a\left(\xi_{Y i}, \zeta\right)\right)
\end{aligned}
$$

Here $Y_{i}$ is a vector whose $i$ :th component is $y_{i}^{\left(r_{i}\right)}$ and whose other components are zero, while $\xi_{Y i}$ is the vector which is obtained when $\xi_{k}^{i}, k=1, \ldots, r_{i}$ is replaced by $y_{i}^{(k-1)}$, and all other components are zeroed.

Proof. The control

$$
u=\left(Y_{i}-a\left(\xi_{Y i}, \zeta\right)\right)
$$

substituted into (15) will keep $\xi_{i}^{k}=0, k \neq i$ provided their initial conditions are zero, and will consequently keep $y_{k}=0, k \neq i$, while we get $\xi_{j}^{i}=y_{i}^{(j)}$. (This is the usual inversion property.) Substituting the $j$ :th component of this vector into (15) while zeroing the other components of $u$ gives the desired result.

The dynamic system described by this proposition can be fairly complex. The physical interpretation is that the extent to which $\tilde{y}_{i}$ and $y_{i}$ differ is a measure of the interaction from other control loops while controlling $y_{i}$ from $u_{j}$. There is however one calculation which is easy to make.

Proposition 3 The direct dependence between $\tilde{y}$ and $y$ in (17), (18) has the form

$$
\tilde{y}_{i}^{\left(r_{i}\right)}=R(\xi, \eta)_{i j} R\left(\xi_{Y i}, \zeta\right)_{j i}^{-1} y_{i}^{\left(r_{i}\right)}+r
$$

where $r$ depends on the state of (17), (18) and lower order derivatives of $y_{i}$.

Proof. This follows by substituting the expression for $u_{j}$ into (17).

From this proposition we see that the matrix

$$
R . * R^{-T}
$$

formed from the decoupling matrix in analogy with (1), (with the elements of $R$ evaluated at appropriate values of the state) can be interpreted as a measure of the "high frequency" interaction in the system. This complements the steady state approach of section 2 .

Example 2 Consider again example 1. Evaluating the decoupling matrix $R$ at some stationary point $x^{0}$ gives

$$
R\left(x^{0}\right)=\left[\begin{array}{cc}
0 & \alpha \\
\frac{\beta}{2 \sqrt{x_{2}^{0}}} & \frac{1}{2 \sqrt{x_{4}^{0}}}
\end{array}\right]
$$

so that (20) becomes

$$
\left[\begin{array}{ll}
0 & 1 \\
1 & 0
\end{array}\right]
$$

As expected from the physics, the high frequency dependence is from $u_{1}$ to $y_{2}$ and from $u_{2}$ to $y_{1}$. 


\section{Conclusions}

We have discussed some possible extensions of the RGA to nonlinear systems. There is a huge literature on the significance of the linear RGA for handling various control problems. The corresponding nonlinear work remains to be done.

\section{Acknowledgment}

This work was supported by the Swedish Research Council for Engineering Sciences (TFR), which is gratefully acknowledged.

\section{References}

[1] E. H. Bristol. On a new measure of interactions for multivariable process control. IEEE Transactions on Automatic Control, AC-11:133-134, (1966).

[2] P. J. Campo and M. Morari. Achievable closed-loop properties of systems under decentralized control: Conditions involving the steady-state gain. IEEE Transactions on Automatic Control, 39:932-943, (1994).

[3] R. M. Hirschorn. Invertibility of multivariable nonlinear control systems. IEEE Transactions on Automatic Control, AC-24(6):855-865, (1979).

[4] M. Hovd and S. Skogestad. Simple frequency-dependent tools for control system analysis, structure selection, and design. Automatica, 28:989-996, (1992).

[5] A. Isidori. Nonlinear Control Systems. Springer-Verlag, New York, (1989).

[6] Sahjendra N. Singh. Decoupling of invertible nonlinear systems with state feedback and precompensation. IEEE Transactions on Automatic Control, AC-25(6):1237-1239, (1980).

[7] Sahjendra N. Singh. A modified algorithm for invertibility of nonlinear systems. IEEE Transactions on Automatic Control, AC-26(2):595-598, (1981). 